\title{
Dust-enshrouded AGNs Can Dominate Host-galaxy-scale Cold Dust Emission
}

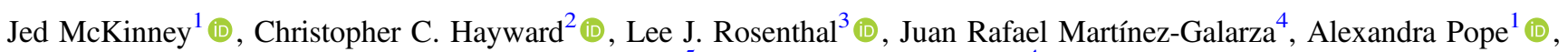 \\ Anna Sajina ${ }^{5}$ (D), and Howard A. Smith ${ }^{4}$ \\ ${ }^{1}$ Department of Astronomy, University of Massachusetts, Amherst, MA 01003, USA; jhmckinney@umass.edu \\ ${ }^{2}$ Center for Computational Astrophysics, Flatiron Institute, 162 Fifth Avenue, New York, NY 10010, USA \\ ${ }^{3}$ Department of Astronomy, California Institute of Technology, Pasadena, CA 91125, USA \\ ${ }^{4}$ Center for Astrophysics | Harvard \& Smithsonian, 60 Garden Street, Cambridge, MA 02138, USA \\ ${ }^{5}$ Department of Physics \& Astronomy, Tufts University, Medford, MA 02155, USA \\ Received 2021 March 22; revised 2021 July 26; accepted 2021 July 26; published 2021 October 29
}

\begin{abstract}
It is widely assumed that long-wavelength infrared (IR) emission from cold dust ( $T \sim 20-40 \mathrm{~K})$ is a reliable tracer of star formation even in the presence of a bright active galactic nucleus (AGN). Based on radiative transfer (RT) models of clumpy AGN tori, hot dust emission from the torus contributes negligibly to the galaxy spectral energy distribution (SED) at $\lambda \gtrsim 100 \mu \mathrm{m}$. However, these models do not include AGN heating of host-galaxy-scale diffuse dust, which may have far-IR (FIR) colors comparable to cold diffuse dust heated by stars. To quantify the contribution of AGN heating to host-galaxy-scale cold dust emission at $\lambda \gtrsim 100 \mu \mathrm{m}$, we perform dust RT calculations on a simulated galaxy merger both including and excluding the bright AGN that it hosts. By differencing the SEDs yielded by RT calculations with and without AGNs that are otherwise identical, we quantify the FIR cold dust emission arising solely from reprocessed AGN photons. In extreme cases, AGN-heated hostgalaxy-scale dust can increase galaxy-integrated FIR flux densities by factors of 2-4; star formation rates calculated from the FIR luminosity assuming no AGN contribution can overestimate the true value by comparable factors. Because the FIR colors of such systems are similar to those of purely star-forming galaxies and redder than torus models, broadband SED decomposition may be insufficient for disentangling the contributions of stars and heavily dust-enshrouded AGNs in the most IR-luminous galaxies. We demonstrate how kiloparsec-scale resolved observations can be used to identify deeply dust-enshrouded AGNs with cool FIR colors when spectroscopic and/ or X-ray detection methods are unavailable.
\end{abstract}

Unified Astronomy Thesaurus concepts: Infrared galaxies (790); AGN host galaxies (2017); Galaxy evolution (594); Hydrodynamical simulations (767); Radiative transfer simulations (1967); Ultraluminous infrared galaxies (1735)

\section{Introduction}

Most of the stellar mass in galaxies was assembled between a redshift of $z=3$ and 1 (e.g., Carilli \& Walter 2013; Madau \& Dickinson 2014); at $z=2$, the star formation rate (SFR) density of the universe peaked, fueled by enhanced gas accretion from the intergalactic medium (Kereš et al. 2005, 2009; Genzel et al. 2008; Tacconi et al. 2010). Statistical analysis of X-ray and infrared (IR) observations of galaxies hosting active galactic nuclei (AGNs) supports the coincident mass growth of central supermassive black holes (SMBHs; Shankar et al. 2009; Aird et al. 2010; Delvecchio et al. 2014) and possibly SMBH - galaxy coevolution (e.g., Ferrarese \& Merritt 2000; Gebhardt et al. 2000; Kormendy \& Ho 2013; Madau \& Dickinson 2014). In particular, AGN feedback may be responsible for quenching star formation (e.g., Springel 2005; Cicone et al. 2014) and/or maintaining quenched galaxies by suppressing cooling flows (e.g., Kereš et al. 2005; Bower et al. 2006; Croton et al. 2006). AGN feedback helps cosmological theories of galaxy evolution match observations-in particular, the number counts of massive and quenched galaxies at $z=0$ (e.g., Bower et al. 2006; Nelson et al. 2018).

Testing this framework against observations, particularly at high redshift, is limited by the ability to separate the AGN and star-forming components of the spectral energy distributions (SEDs) of galaxies, as to test the "quasar mode" of AGN feedback it is necessary to simultaneously constrain the AGN luminosity and SFR at a time when the AGN should be heavily dust enshrouded (e.g., Di Matteo et al. 2005; Hopkins et al. 2008). The far-IR (FIR; $\lambda \gtrsim 100 \mu \mathrm{m}$ )/submillimeter regime is thus a powerful probe of dust-obscured mass assembly. It has long been known that dust-enshrouded AGNs can dominate the total IR luminosity, powered predominantly by warm dust emitting at $5 \mu \mathrm{m}<\lambda_{\text {rest }} \leqslant 30 \mu \mathrm{m}$ (e.g., Sanders et al. 1988), but it is typically assumed that AGNs contribute negligibly to FIR emission at wavelengths longer than rest frame $100 \mu \mathrm{m}$, as predicted by torus models (e.g., Fritz et al. 2006; Nenkova et al. 2008a, 2008b). Consequently, cold dust emission at rest-frame wavelengths $\gtrsim 100 \mu \mathrm{m}$ is sometimes treated as a "safe" SFR tracer even when an AGN dominates the bolometric luminosity of the galaxy by explicitly converting the FIR luminosity into an SFR value (e.g., Hatziminaoglou et al. 2010; Kalfountzou et al. 2014; Azadi et al. 2015; Gürkan et al. 2015; Banerji et al. 2018; Stacey et al. 2018; Wethers et al. 2020).

Alternatively, the full SED may be fit using an SED modeling code that includes an AGN component (e.g., Chang et al. 2017; Ciesla et al. 2015; Lanzuisi et al. 2017; Dietrich et al. 2018; Leja et al. 2018; Pouliasis et al. 2020; Ramos Padilla et al. 2020; Yang et al. 2020). Such SED modeling codes generally assume that AGNs contribute negligibly to cold dust emission longward of $\sim 100 \mu \mathrm{m}$ and rely on SED model libraries generated by performing radiative transfer (RT) on AGN torus models. The resulting SEDs include hot dust emission from the torus but not potential AGN-powered cold dust emission on host-galaxy scales by construction, even 
though UV/optical photons emitted by the accretion disk or IR photons from the torus can in principle heat interstellar medium (ISM) dust directly and/or by progressive attenuation and reradiation at longer wavelengths. That this possibility is realized in some systems is suggested by results indicating that the host galaxy can be responsible for a nonnegligible fraction of the obscuring column density in some AGNs (Hickox \& Alexander 2018 and references therein).

The origin of the FIR emission in galaxies hosting luminous AGNs has been debated over the past few decades (e.g., Downes \& Solomon 1998; Page et al. 2001; Franceschini et al. 2003; Ruiz et al. 2007; Kirkpatrick et al. 2012, 2015). Over the years, a growing body of literature has been making the case for AGN-powered FIR emission at wavelengths $\gtrsim 100 \mu \mathrm{m}$ in QSOs (e.g., Sanders et al. 1989; Yun \& Scoville 1998; Nandra \& Iwasawa 2007; Petric et al. 2015; Schneider et al. 2015; Symeonidis et al. 2016; Symeonidis 2017; Symeonidis \& Page 2018); however, other works find conflicting results (e.g., Stanley et al. 2017; Shangguan et al. 2020; DiMascia et al. 2021). Spatially resolved observations of heavily obscured nearby systems find that a significant fraction of the galaxyintegrated FIR/submillimeter emission can originate from very small regions (e.g., $\lesssim 15$ pc for Arp 220; Scoville et al. 2017), suggestive that deeply dust-obscured AGNs power this emission (as it is unlikely that a star-forming region forming stars at a rate $\gtrsim 100 M_{\odot} \mathrm{yr}^{-1}$ would be this compact). However, the fact that these nuclear regions can be opaque well into the FIR makes it difficult to conclusively identify the power source, and how to infer the amount of host-galaxy, kiloparsecscale dust heating due to AGN emission is unclear.

A promising method for decomposing galaxy-integrated FIR SEDs into AGN and star-forming components directly relies on mid-IR spectroscopy (e.g., Pope et al. 2008). Kirkpatrick et al. (2012, 2015) use this technique to subtract AGN-heated dust from the SEDs of a sample of high- $z$ dusty galaxies using midIR spectroscopic methods assuming that all of the $\sim 80-90 \mathrm{~K}$ dust emission is attributed to the AGN alone. These authors find that up to $75 \%$ of $L_{\mathrm{IR}}$ can be powered by AGN heating of the hot dust in galaxies with spectroscopic signatures of AGNs in the mid-IR. Whether this conclusion extends to host-galaxyscale cold dust emission at wavelengths past $100 \mu \mathrm{m}$ is difficult to test empirically because of sparse wavelength coverage in the rest-frame FIR/submillimeter and because dust reprocessing erases information about the original energy source. Roebuck et al. (2016) applied Kirkpatrick et al.'s method to attempt to recover the AGN fraction of simulated galaxies, including the one employed in this work. Although the recovered and true AGN fractions were in qualitative agreement, Roebuck et al. (2016) found that SED decomposition underestimated the AGN contribution to the IR luminosity in some cases.

In this work, we build on previous work in which we used hydrodynamical simulations plus RT calculations to investigate the effects of galaxy-scale dust reprocessing of AGN torus emission (Younger et al. 2009; Snyder et al. 2013; Roebuck et al. 2016). Specifically, we use a hydrodynamical simulation of an equal-mass galaxy merger post-processed with RT to investigate the possibility that heating from a luminous AGN embedded within a dusty galaxy can power host-galaxy-scale cold dust emission at rest-frame wavelengths $\lambda \gtrsim 100 \mu \mathrm{m}$, well beyond the shorter-wavelength regime in which significant AGN powering of dust continuum flux densities is well accepted by the community (Sanders et al. 1988; Casey et al. 2014). We create initial conditions for two identical galaxies on a parabolic orbit. The hydrodynamical simulation evolves the system, including models for star formation, stellar feedback, black hole accretion, and AGN feedback. Then, we perform dust RT calculations in post-processing for various time snapshots to compute spatially resolved UV-millimeter SEDs of the simulated galaxy merger. By performing dust RT calculations both including and excluding the emission from the AGN, we can explicitly identify IR emission powered by the AGN on both torus and host-galaxy (kiloparsec) scales. This particular simulation is a massive, gas-rich merger designed to be analogous to $z \sim 2-3$ submillimeter galaxies (SMGs), and it is indeed consistent with many properties of observed SMGs (Hayward et al. 2011, 2012, 2013). We chose this particular simulation because it exhibits a high SFR, a high AGN luminosity, and high dust attenuation; such systems are the most likely candidates for AGNs powering host-galaxyscale cold dust emission. We show that in this admittedly extreme system, dust-enshrouded AGNs can be the dominant power source of cold dust emission on host-galaxy scales, with cool FIR colors indistinguishable from those of purely starforming galaxies. We estimate the potential bias in observations of galaxy SFRs and discuss implications for SED decomposition.

The remainder of this paper is organized as follows: In Section 2, we discuss the details of our numerical simulation and RT calculations. Section 3 explains how we extract the AGN-powered dust emission and presents our main results. We discuss the implications of these calculations for inferred trends in galaxy evolution and limitations of this work in Section 4. We summarize in Section 5. Throughout this work, we assume a Salpeter initial mass function and adopt a $\Lambda$ CDM cosmology with $\Omega_{m}=0.3, \Omega_{\Lambda}=0.7$, and $H_{0}=70 \mathrm{~km} \mathrm{~s}^{-1} \mathrm{Mpc}^{-1}$.

\section{Simulation and Radiative Transfer Details}

This work makes use of the results of RT calculations originally presented in Snyder et al. (2013). Our reanalysis focuses on the output from a Gadget-2 simulation (Springel 2005) of an idealized (noncosmological) major merger of two identical disk galaxies. The initial conditions were generated following Springel et al. (2005). The initial halo and baryonic masses are $9 \times 10^{12} M_{\odot}$ and $4 \times 10^{11} M_{\odot}$, respectively. The initial black hole mass is $1.4 \times 10^{5} M_{\odot}$, and the initial gas fraction is $60 \%$. Star formation and stellar feedback are modeled as described in Springel \& Hernquist (2003) and Springel et al. (2005). Black holes grow via Eddington-limited Bondi-Hoyle accretion.

The RT code SUNRISE (Jonsson 2006; Jonsson et al. 2010) was used to compute SEDs for seven viewing angles every $10 \mathrm{Myr}$ throughout the simulation run. The outputs of the hydrodynamical simulation are used as input for the RT calculations, i.e., the former is used to specify the $3 \mathrm{D}$ distribution of sources of emission (stars and AGNs) and dust. The ages and metallicities of the star particles are used to assign single-age stellar population SEDs to individual star particles. The metal distribution determines the dust density distribution; a dust-to-metals ratio of 0.4 was assumed. For more in-depth discussion of the simulation assumptions, subgrid models, and numerical methods, see Snyder et al. (2013) and Hayward et al. (2011). We stress that while the subgrid models employed in this work are admittedly limited approximations of reality, they 
have been tested in previous works and are found to yield galaxies with properties broadly consistent with high- $z$ dustobscured galaxies. With this in mind, this study should be considered a somewhat idealized numerical experiment designed to cleanly identify reprocessed dust emission powered by AGNs in a "reasonable" simulated merger rather than an attempt to perfectly reproduce reality.

For each time snapshot of the merger simulation, different RT calculations were performed; they differ only in how the luminosity of the AGN is computed. The "fiducial" RT runs use the accretion rate from the simulation to compute the AGN luminosity, assuming $10 \%$ radiative efficiency. We use luminosity-dependent AGN SED templates derived from observations of unreddened QSOs (Hopkins et al. 2007) as the input SEDs emitted by the AGN particle(s); see Snyder et al. (2013) for details. In the "AGN-off" runs, the luminosity of the AGN is artificially set to zero (i.e., only stellar emission is considered in the RT calculations). We emphasize that AGN feedback is still included because the same hydrodynamical simulation is used as input, so these runs determine the impact of the AGN emission on the total SED all else being equal, including feedback from the AGN on the host-galaxy ISM. Finally, in the "AGN-10x" RT calculations, the luminosity of the AGN is artificially boosted by a factor of 10 (equivalent to assuming a radiative efficiency of $100 \%$ or an instantaneous accretion rate 10 times the value computed in the simulation). As in the "AGN-off" runs, the AGN feedback is not altered (the same hydrodynamical simulations are used) because we wish to isolate the effect of the AGN on the SED. In other words, the input of a boosted AGN spectrum into the RT calculations is the only difference between the AGN-10x and AGN-off results. Notably, this technique ignores how increasing the AGN luminosity by a factor of 10 would affect the evolution of the system; this is by design, as to extract the AGN-powered IR emission we need all other aspects of the run (e.g., the dust geometry and SFR) to be fixed.

Snyder et al. (2013) performed these calculations simply to span a larger range in the AGN fractional contribution to the bolometric luminosity, but these should not be considered "unphysical" by any means, as the relatively crude accretion model employed and the limited resolution of the hydrodynamical simulation may cause the $\mathrm{BH}$ accretion rate to be underestimated (e.g., Hayward et al. 2014; Angles-Alcazar et al. 2021). The Eddington ratio $\left(\lambda_{\text {edd }} \equiv L_{\mathrm{AGN}} / L_{\text {edd }}\right)$ varies from 0 to 1 in the fiducial runs and thus spans a range of $\lambda_{\text {edd }}=0-10$ in the AGN-10x runs. There is both theoretical (e.g., Begelman 2002; Jiang et al. 2014) and observational (e.g., Kelly \& Shen 2013; Shirakata et al. 2019) evidence for super-Eddington accretion.

\section{Results}

For every snapshot and each of the seven viewing angles, we calculate the SED of the AGN-powered dust emission, i.e., the dust emission implicit in the input torus model SED plus the host-galaxy dust emission powered by the AGN (rather than stars). Our SUNRISE calculations with and without AGN emission are otherwise identical; therefore, taking the difference between the SEDs with and without AGN emission yields the SED of all photons of AGN origin, including those reprocessed by dust. Originally demonstrated in Roebuck et al. (2016) for our fiducial simulation (see their Figure 2), this differencing technique is shown in Figure 1 for the AGN-10x

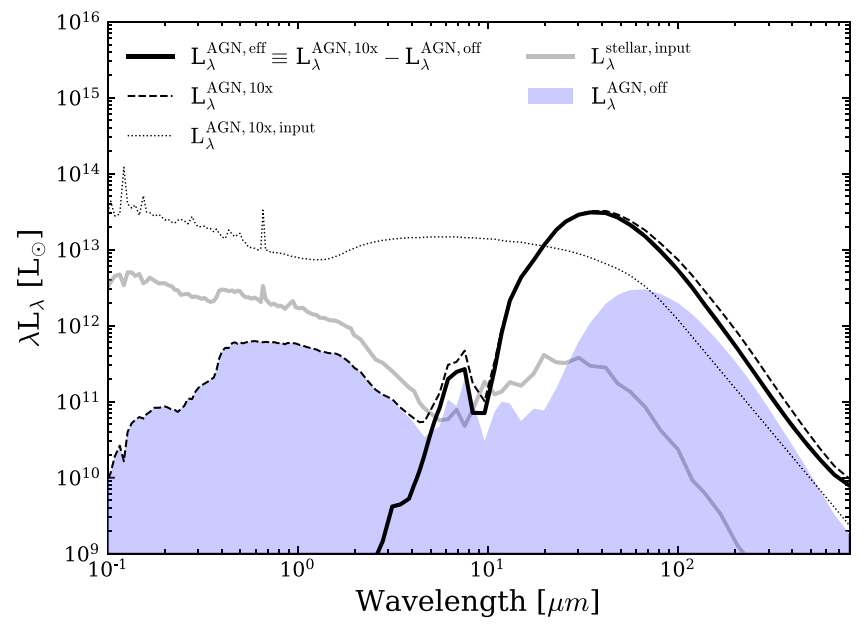

Figure 1. Rest-frame SED for the AGN-10x model (dashed line) at the time of peak $L_{\mathrm{AGN}} / L_{\mathrm{SF}}$, where the AGN dominates the bolometric luminosity and the time at which the AGN contributes maximally to the FIR luminosity during this simulation. The unattenuated AGN torus spectrum $\left(L_{\lambda}^{\mathrm{AGN}, 10 \mathrm{x} \text {,input }}\right)$ is shown as a dotted line, and the unattenuated stellar spectrum is shown as a solid gray line $\left(L_{\lambda}^{\text {stellar,input }}\right)$. For the same simulation snapshot, we show the corresponding AGN-off SED shaded in blue. The effective AGN SED, calculated by taking the difference between the AGN-10x and AGN-off SEDs, is indicated by the solid line. This corresponds to the primary torus emission attenuated by dust plus host-galaxy-scale thermal dust emission powered by the AGN. Due to heavy dust obscuration experienced by the AGN, the AGN contributes negligibly to the UV-optical SED, but it dominates longward of a few microns, including the FIR cold dust emission traditionally associated with star formation.

run at $t_{\text {peak }}$, the simulation time when the ratio of bolometric AGN luminosity $\left(L_{\mathrm{AGN}}\right)$ to bolometric stellar luminosity $\left(L_{\mathrm{SF}}\right)$ reaches its maximal value of $\sim 10$. At this time, the mass of the $\mathrm{BH}$ is $3.2 \times 10^{8} M_{\odot}$, and it is accreting at a rate of $82 M_{\odot} \mathrm{yr}^{-1}$, while $\mathrm{SFR}=470 M_{\odot} \mathrm{yr}^{-1}$. The SEDs shown in Figure 1 are the following:

1. $L_{\lambda}^{\mathrm{AGN}, 10 \mathrm{x}}$.- The attenuated+reradiated SED corresponding to the AGN-10x run, where the luminosity of the AGN is artificially boosted by a factor of 10 .

2. $L_{\lambda}^{\mathrm{AGN}, \text { off }}$.- The attenuated + reradiated SED corresponding to the RT run where the luminosity of the AGN is set to zero.

3. $L_{\lambda}^{\mathrm{AGN}, \mathrm{eff}}$.-The "effective AGN SED" calculated from the

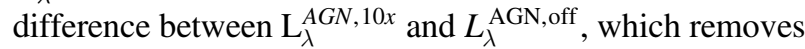
stellar-heated dust emission, leaving behind only AGN photons, including IR photons from the torus that are absorbed by dust in the ISM and reradiated at progressively longer wavelengths.

4. $L_{\lambda}^{\mathrm{AGN}, 10 \mathrm{x} \text {,input }}$-The input AGN SED (from Hopkins et al. 2007) in the AGN-10x run. This SED is integrated from 0.1 to $1000 \mu \mathrm{m}$ to calculate $L_{\mathrm{AGN}}$.

5. $\mathrm{L}_{\lambda}^{\text {stellar,input }}$.-The unattenuated stellar spectrum, which is the same across all RT runs. This SED is integrated over $0.1-1000 \mu \mathrm{m}$ to determine $L_{\mathrm{SF}}$.

$L_{\lambda}^{\text {AGN, eff }}$ peaks at $31 \mu \mathrm{m}$, corresponding to an effective dust temperature (from Wien's law) of $96 \mathrm{~K}$, on the warm end of dust temperatures observed in AGN host galaxies (Kirkpatrick et al. 2015). In the simulated SEDs, most of the IR emission between 10 and $50 \mu \mathrm{m}$ comes from reprocessed AGN photons, consistent with numerous observations of local and high- $z$ ultraluminous infrared galaxies (ULIRGs; $L_{\mathrm{IR}}>10^{12} L_{\odot}$ ) hosting AGNs (e.g., Genzel et al. 1998; Sajina et al. 2007). 


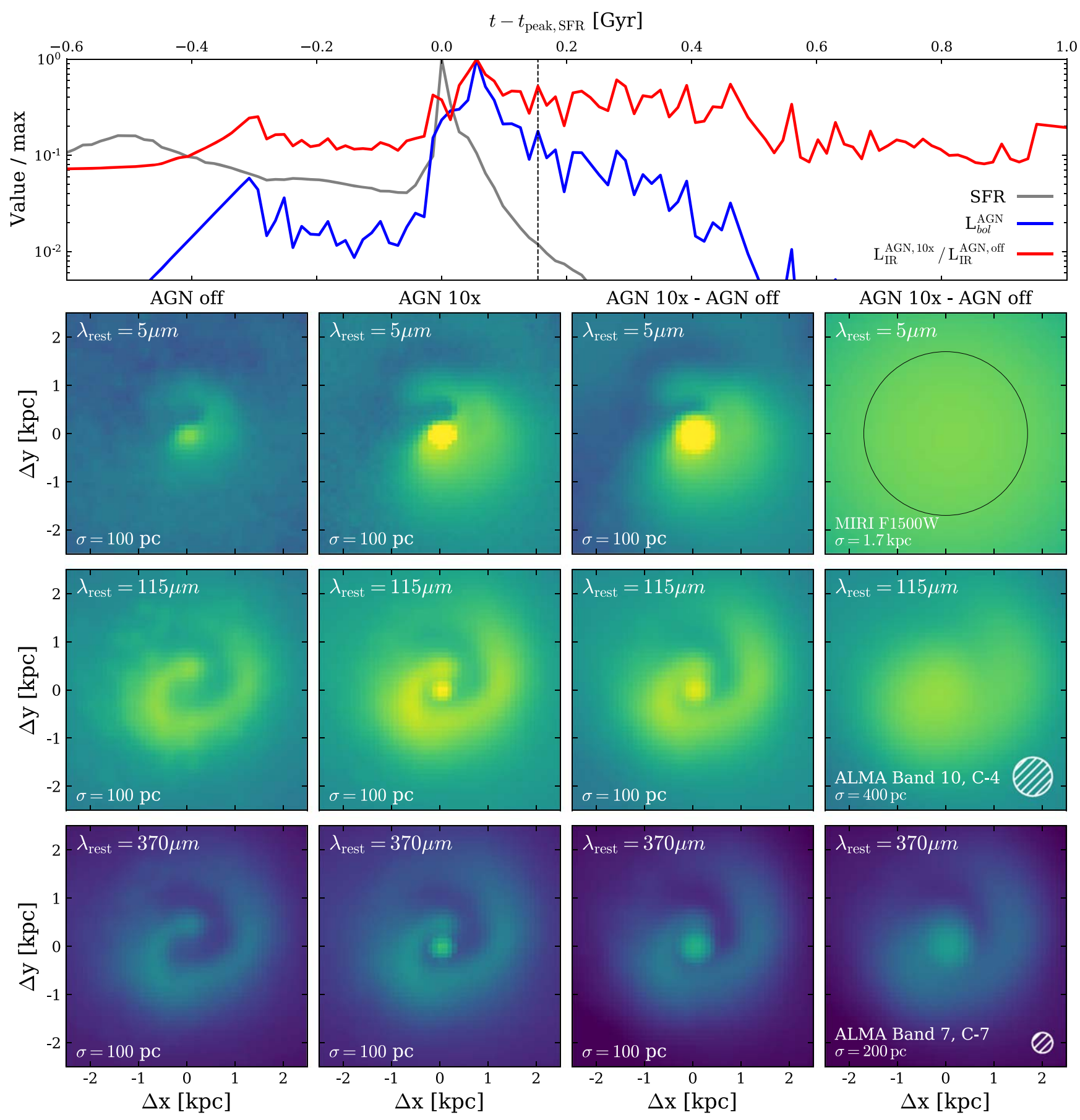

Figure 2. Emission maps of the simulated merger at a representative time (marked by the dashed line in the top panel) following the peak SFR and peak bolometric AGN luminosity. The first (second) column shows maps of the emission at rest-frame wavelengths $15 \mu \mathrm{m}, 115 \mu \mathrm{m}$, and $370 \mu \mathrm{m}$ for the AGN-off (AGN-10x) RT run. The third column shows difference images between the AGN-10x and AGN-off maps, i.e., the dust emission powered directly by the AGN. The color scale and stretch are constant across. For comparison with observations, we blur the images to $100 \mathrm{pc}$ resolution, typical of spatially resolved studies of lensed systems at $z \sim 2$. In the far-right column, we further blur each map to the spatial resolution achievable with JWST/MIRI (top) and ALMA high-frequency observations in extended array configurations (middle, bottom) for a galaxy at $z=2$. The JWST/MIRI beam is shown as a solid black circle, and the ALMA beams as white hatched circles. The AGN-powered cold dust emission includes both a compact nuclear component and an extended component spanning a few kiloparsecs.

Notably, $L_{\lambda}^{\mathrm{AGN}, \text { eff }}$ is greater than $L_{\lambda}^{\mathrm{AGN}, \text { off }}$ by a factor of $\gtrsim 2$ from $100-1000 \mu \mathrm{m}$. In this particular extreme case, even the coldest dust is predominantly heated by the AGN.

The top panel of Figure 2 shows the time evolution of the SFR, AGN luminosity, and fractional contribution of the AGN to the IR luminosity. Near coalescence of the two galaxies, there is a strong starburst. The SFR then rapidly decays owing to gas consumption (there is no cosmological gas accretion in this idealized simulation) and AGN feedback. Approximately 50 Myr after the peak SFR, the AGN luminosity peaks. During this period, both the newly formed stars and AGNs are deeply obscured by dust. The AGN contributes $\gtrsim 30 \%$ of the IR luminosity for $\sim 0.5 \mathrm{Gyr}$, starting at the peak of the starburst. The AGN luminosity then declines, decreasing below $10 \%$ of its peak luminosity $\sim 100 \mathrm{Myr}$ later. 


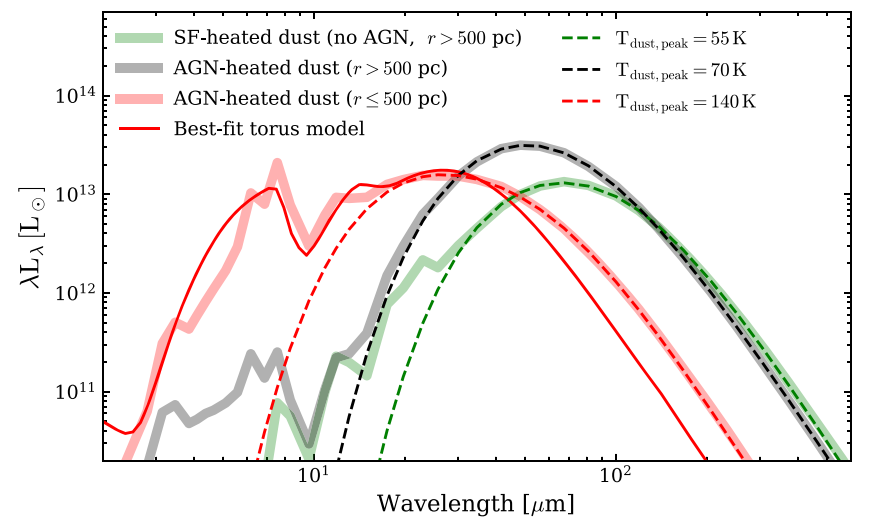

Figure 3. Rest-frame SED of AGN-heated dust emission including direct heating and heating from dust self-absorption in the central kiloparsec of the simulation (red, $r \leqslant 500 \mathrm{pc}$ ) and in the extranuclear region (black, $r>500 \mathrm{pc}$ ), both extracted from the difference map (i.e., Figure 2, third column), $\sim 150 \mathrm{Myr}$ after $t_{\text {peak }}$. The green SED corresponds to diffuse star-formationheated dust at $r>500 \mathrm{pc}$ in the AGN-off run (Figure 2, first column). Dashed lines show modified blackbody models that best fit the $r>500$ pc SEDs at $\lambda>10 \mu \mathrm{m}$. The solid red line shows the best-fit dusty, AGN torus model from the Fritz et al. (2006) library to the AGN-heated dust within the central kiloparsec, which exhibits $T_{\text {dust }}=140 \mathrm{~K}$ from Wien's law. The AGN-heated dust emission within the central kiloparsec is dominated by the hot, dusty torus, whereas the diffuse extended component is well described by a modified blackbody with $T_{\text {dust }}=70 \mathrm{~K}$, warmer than the star-formation-heated diffuse dust, but cooler than the torus emission.

The lower part of Figure 2 presents maps of the 5, 115, and $370 \mu \mathrm{m}$ dust emission in the AGN-off (first column) and AGN$10 \mathrm{x}$ (second column) runs $\sim 150 \mathrm{Myr}$ after $t_{\text {peak }}$, a time at which there is significant dust-obscured star formation but the dustenshrouded AGN dominates the luminosity $\left(L_{\mathrm{AGN}} / L_{\mathrm{SF}}>1\right)$; this time is marked by the dashed vertical line in the top panel. Given the short duration of the starburst and peak in AGN luminosity, most observations of heavily dust-obscured AGNs should probe this phase (subsequent to the peak SFR and AGN luminosity), so these maps should be considered "representative." Figure 2 also shows the difference between the AGN-10x and AGN-off simulations (third column), which captures the spatial extent of the dust emission powered by the AGN. These maps have been convolved with a Gaussian with FWHM of $100 \mathrm{pc}$, representative of spatially resolved studies of lensed systems at $z \sim 2-3$ (e.g., Swinbank et al. 2015; Cañameras et al. 2017; Massardi et al. 2018; Sharda et al. 2018).

The third column of Figure 2 shows that the cold dust emission at FIR wavelengths $\gtrsim 100 \mu \mathrm{m}$ powered by the AGN consists of a compact nuclear component and a lower surface brightness extended component on kiloparsec scales, clearly demonstrating that photons from the AGN heat cold dust throughout the host galaxy on kiloparsec scales. Indeed, the integrated emission within the central kiloparsec is well fit by a hot, dusty torus model from the Fritz et al. (2006) library, as shown in Figure 3. On the other hand, the host-galaxy-scale AGN-heated diffuse dust is well fit by a modified blackbody with $T_{\text {dust }}=70 \mathrm{~K}$, warmer than the kiloparsec-scale starformation-heated dust by $15 \mathrm{~K}$, but significantly cooler than the dusty torus emission within the central kiloparsec. Notably, $22 \%(78 \%)$ of the reprocessed AGN luminosity between 8 and $1000 \mu \mathrm{m}(100-500 \mu \mathrm{m})$ arises from outside the central kiloparsec. The innermost region powers $\sim 25 \%$ of $L_{\mathrm{IR}}$ (accounting for star formation and AGN heating) integrated across the map. Comparable fractions of $L_{\mathrm{IR}}$ arising from the centers of galaxies have been measured in nearby systems with spatially resolved

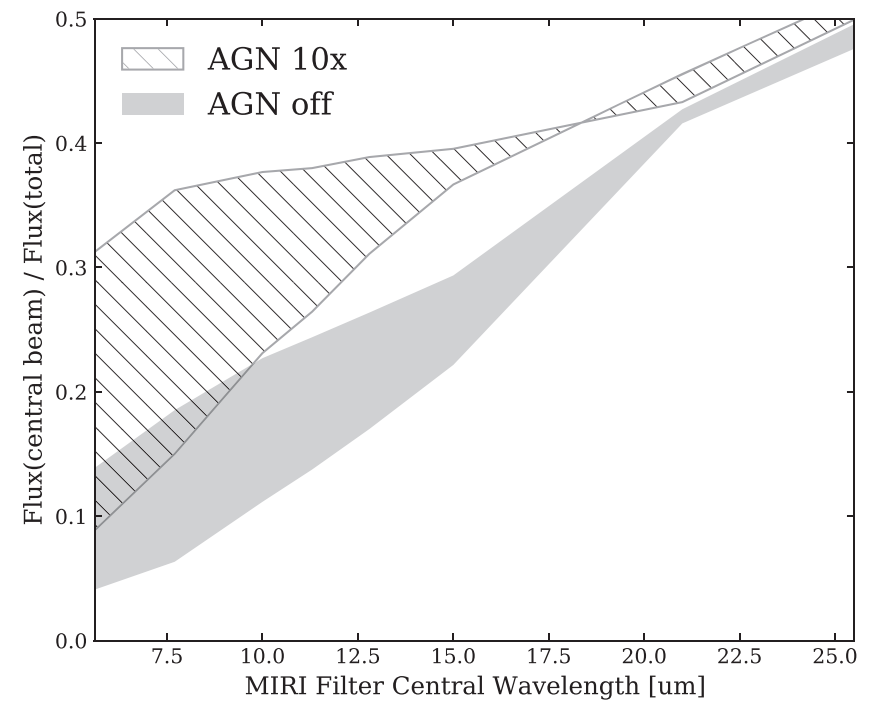

Figure 4. The ratio of flux within a simulated JWST/MIRI beam placed on the center of the galaxy relative to the total flux across the point-spread-functionconvolved map, for various MIRI filters. We show the results of such mock observations for the AGN-10x (hatched) and AGN-off (solid gray) runs at $t_{\text {peak }}$, the same snapshot used in Figure 2. The maps have been redshifted to $z=2$ and convolved with the wavelength-dependent point-spread function of JWST/ MIRI. The shaded and hatched regions contain the range of simulated observations for all seven viewing angles. The presence of a powerful, dustenshrouded AGN boosts the fraction of the total flux within the central beam by a factor of $\sim 1.5$ between observed wavelengths of $\sim 6$ and $15 \mu \mathrm{m}$. Mid-IR imaging at JWST's resolution could be used to identify such dustenshrouded AGNs.

FIR observations such as in the west nucleus of Arp 220 (Scoville et al. 2017).

In the fourth column of Figure 2, we show maps of the AGN-powered dust emission convolved to the spatial resolution that JWST/MIRI (top) and ALMA's high-frequency, extended configurations (middle, bottom) can achieve for an unlensed galaxy at $z=2$. The nuclear dust heated by the dustenshrouded AGN can be resolved from the extended cold dust with ALMA. At coarser spatial resolutions, imaging with JWST/MIRI can test for buried AGN heating host-galaxy dust by comparing central and extended emission on scales within and outside $\sim 2 \mathrm{kpc}$ (solid circle), nearly twice the $1.1 \mathrm{kpc}$ halfmass radius of the system. In Figure 4, we show the ratio of the flux within a simulated JWST/MIRI beam placed on the center of a mock observation to the total flux in the map, for MIRI filters with central wavelengths between 5 and $25 \mu \mathrm{m}$. Before the simulated aperture photometry, we first convolve the maps to the spatial resolution of JWST assuming that the galaxy is at $z=2$. The presence of the luminous dust-enshrouded AGN increases the fraction of the total flux within the central region relative to the total flux by a factor of $\sim 1.5$ in most MIRI filters. As expected, the AGN-10x simulation exhibits more concentrated emission in all MIRI filters than the AGN-off run because of the strong, nuclear dust-heating source. IR imaging and spatially resolved SED fitting will be key for identifying such dust-enshrouded AGNs because, as we shall see below, the cold FIR colors would preclude distinguishing this source from a compact nuclear starburst based on the galaxyintegrated broadband FIR SED alone.

Having demonstrated that the AGN can power cold dust emission on host-galaxy scales, we now quantify how the AGN affects the IR luminosity in different bands throughout the simulation. Taking the ratio of $L_{\mathrm{IR}}$ in the AGN-on and AGN- 

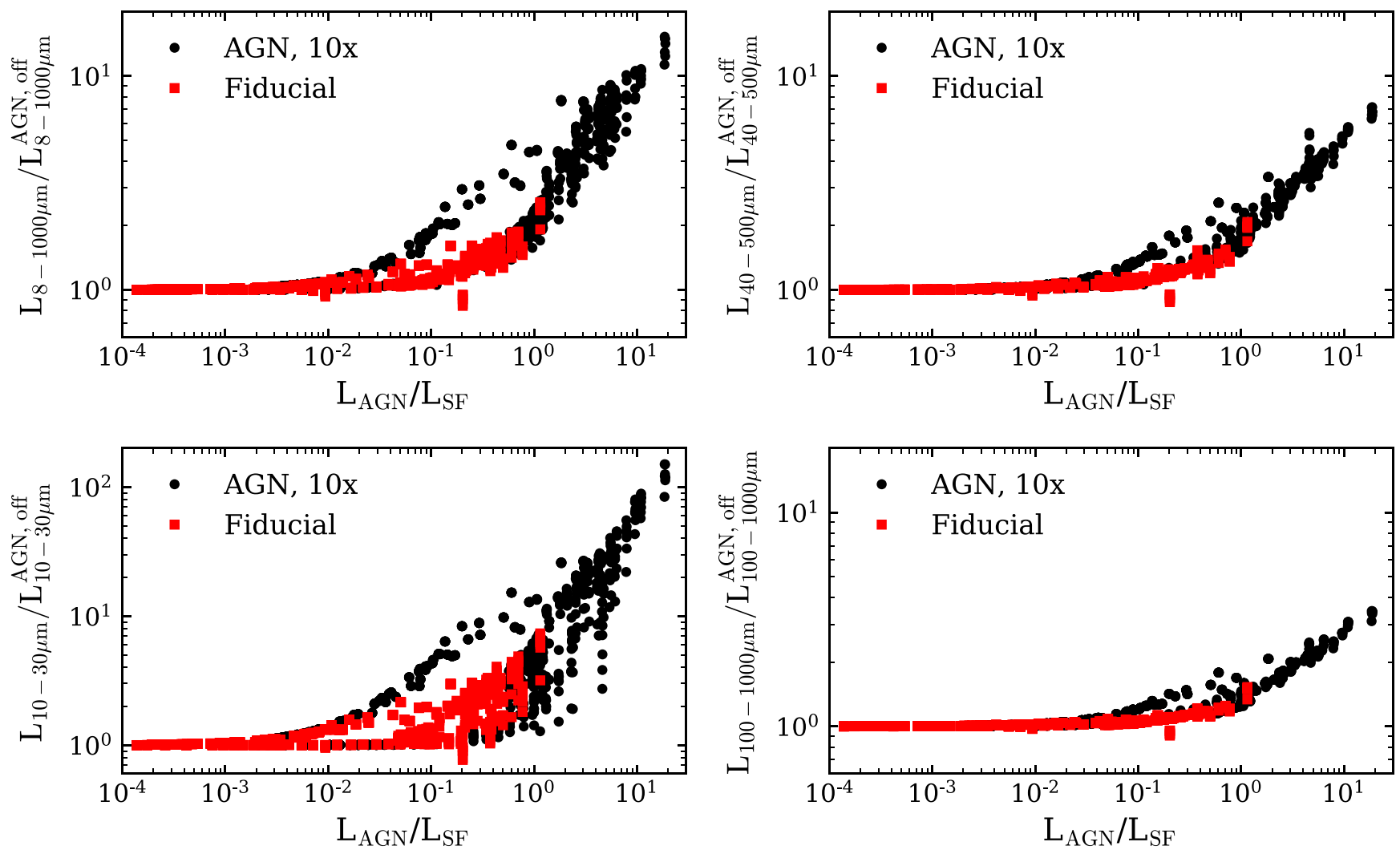

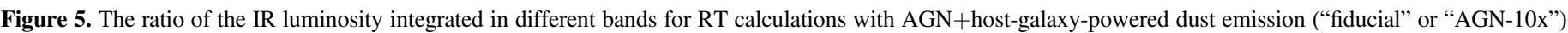

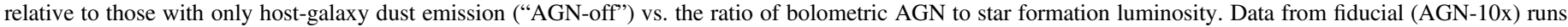

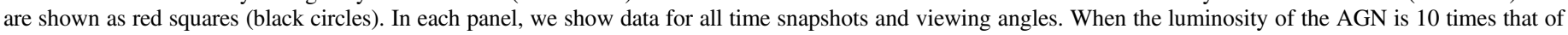

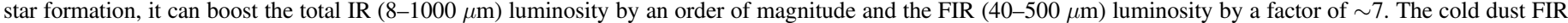

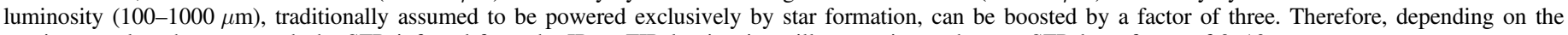
precise wavelength range used, the SFR inferred from the IR or FIR luminosity will overestimate the true SFR by a factor of 3-10.

off simulations captures the fractional change in $L_{\mathrm{IR}}$ due to dust emission powered by photons of AGN origin. In Figure 5, we plot the ratio of integrated IR luminosities between the AGNon and AGN-off simulations in four different wavelength bands versus $L_{\mathrm{AGN}} / L_{\mathrm{SF}}$. The total (far-)IR luminosity from 8 to $1000 \mu \mathrm{m}(40-500 \mu \mathrm{m})$ is boosted by a factor of $\sim 10(\sim 7)$ as $L_{\mathrm{AGN}} / L_{\mathrm{SF}}$ increases from 1 to $\sim 10$. The rise in $L_{\mathrm{IR}}$ with $L_{\mathrm{AGN}} / L_{\mathrm{SF}}$ is mostly driven by increased warm dust emission at shorter wavelengths, as shown in the $10-30 \mu \mathrm{m}$ panel of Figure 5. However, the integrated 100-1000 $\mu \mathrm{m}$ luminosity can be increased by the AGN by as much as a factor of $\sim 3$, demonstrating that even the coldest dust emission can be powered by the AGN. This result is at odds with the notion that cold dust emission at these FIR wavelengths is predominantly heated by young stars even when an AGN dominates the bolometric luminosity of the galaxy (e.g., Stanley et al. 2017; Shangguan et al. 2020).

FIR colors are often used to distinguish AGN-powered and stellar-powered dust emission, as IR-selected galaxies that host AGNs tend to have warmer IR colors than those that do not (e.g., de Grijp et al. 1987; Sanders et al. 1988; but see Younger et al. 2009). For this reason, we present Herschel FIR colorcolor plots of the simulation runs in Figure 6, while also tracking the ratio of $\mathrm{AGN}$ luminosity relative to the star formation luminosity $\left(L_{\mathrm{AGN}} / L_{\mathrm{SF}}\right)$. High $L_{\mathrm{AGN}} / L_{\mathrm{SF}}$ tends to result in bluer colors; however, this is modulated by changes in extinction with time and viewing angle. Higher levels of extinction $\left(A_{V}\right)$ for fixed $L_{\mathrm{AGN}} / L_{\mathrm{SF}}$ yield redder FIR colors. For comparison with observations, we also show the empirically derived galaxy SED templates of Kirkpatrick et al. (2015), which span a range in AGN contribution to the mid-IR emission, as well as the $z \sim 2$ Type 1 and Type 2 AGN sample of Hatziminaoglou et al. (2010). The simulated galaxies have FIR colors on the Wien side of the dust distribution (PACS photometry, Figure 6, top) consistent with the observed galaxies. The SPIRE colors (Figure 6, bottom), which trace the dust peak, of the simulated galaxies are somewhat bluer (warmer) than those of the majority of the observed galaxies by a factor of $\sim 2-3$ when $L_{\mathrm{AGN}} / L_{\mathrm{SF}}>1$, but they are redder (colder) than those of any of the torus models in these two widely used torus SED libraries because the torus models do not include galaxy-scale cold dust emission. These results suggest that the cool FIR colors of the observed AGN hosts are not necessarily evidence that their FIR cold dust emission is powered by ongoing star formation; rather, the AGN itself may be the dominant power source for the cold dust emission.

\section{Discussion \\ 4.1. Implications}

Our simulations suggest that in extreme cases, AGNs may dominate the FIR luminosity of a galaxy at all wavelengths. The central AGN in our simulations heats dust at radii greater than the half-mass radius of the system, on kiloparsec scales, at times following merger coalescence. Moreover, our simulations span a similar parameter space in FIR colors compared to 

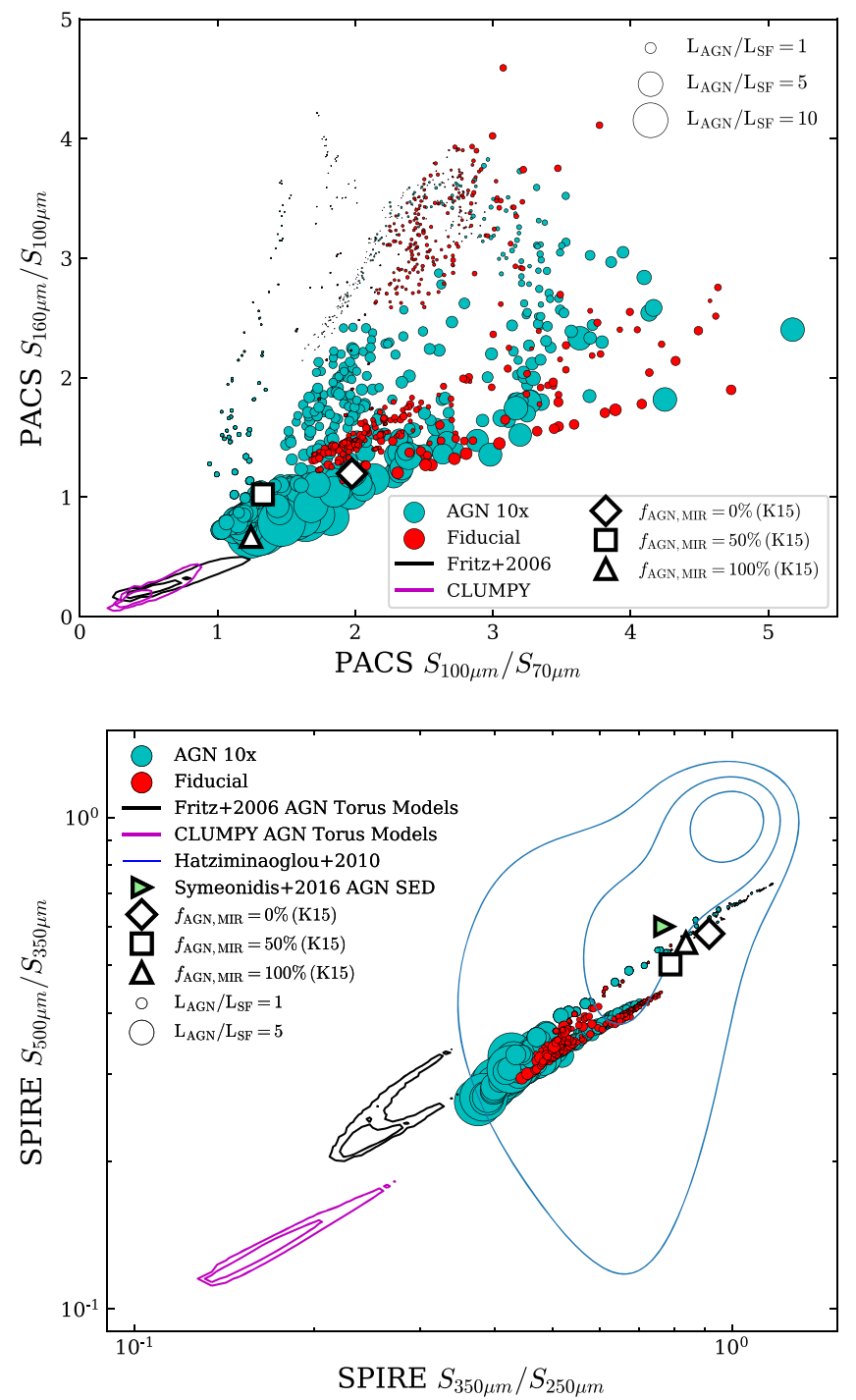

Figure 6. Top: Herschel PACS $100 \mu \mathrm{m} / 70 \mu \mathrm{m}$ vs. $160 \mu \mathrm{m} / 100 \mu \mathrm{m}$ colors (assuming $z=2$ ). Cyan and red points correspond to the AGN-10x and fiducial runs, respectively, and the size of each marker is proportional to $L_{\mathrm{AGN}} / L_{\mathrm{SF}}$. For comparison, we show the colors of empirical templates from Kirkpatrick et al. (2015) (K15) at $f_{\mathrm{AGN}, \mathrm{IR}}=0 \%$ (diamond), $f_{\mathrm{AGN}}$, IR $=50 \%$ (square), and $f_{\mathrm{AGN}}$, IR $=100 \%$ (triangle). Magenta and black contours contain $95 \%$ and $68 \%$ of the distribution in Herschel colors spanned by the Nenkova et al. (2008a, 2008b) and Fritz et al. (2006) dusty AGN torus models, respectively. Bottom: Herschel SPIRE $350 \mu \mathrm{m} / 250 \mu \mathrm{m}$ vs. $500 \mu \mathrm{m} / 350 \mu \mathrm{m}$ colors (assuming $z=2$ ) following the same color scheme as in the top panel. Blue contours contain the $z \sim 2$ AGN-hosting galaxy sample of Hatziminaoglou et al. (2010). Colors from the Kirkpatrick et al. (2015) SEDs are shown as described in the top panel, and we also include the SPIRE colors of the AGN SED from Symeonidis et al. 2016 (green triangle). The FIR colors of the simulated galaxies are redder (cooler) than those of all of the torus models and consistent with those of the observed galaxies, suggesting that the "cold" FIR colors of these AGN hosts are not necessarily indicative of ongoing star formation; instead, the FIR emission may be predominantly powered by dust-enshrouded AGNs.

observations but are redder than all dusty torus models from the CLUMPY (Nenkova et al. 2008a, 2008b) and Fritz et al. (2006) AGN torus SED libraries, instead exhibiting colors that could be interpreted to indicate pure star formation. Consequently, without robust exclusion of a central deeply dust-enshrouded AGN, even cold dust emission at $\gtrsim 100 \mu \mathrm{m}$ may may be "contaminated" by AGN-powered dust emission and thus not yield accurate SFRs.
Table 1

Maximal Boosting of Host-galaxy FIR Dust Emission by a Dust-enshrouded AGN at Selected Wavelengths, and for Integrated IR Luminosities

\begin{tabular}{lcccc}
\hline \hline$\lambda_{\text {obs }}$ & $z=0$ & $z=1$ & $z=2$ & $z=3$ \\
\hline $70 \mu \mathrm{m}$ & 3.8 & 10.8 & 30.9 & 47.4 \\
$100 \mu \mathrm{m}$ & 2.9 & 5.8 & 11.9 & 25.0 \\
$160 \mu \mathrm{m}$ & 2.4 & 3.4 & 5.2 & 8.5 \\
$250 \mu \mathrm{m}$ & 2.2 & 2.6 & 3.3 & 4.3 \\
$350 \mu \mathrm{m}$ & 2.3 & 2.3 & 2.7 & 3.2 \\
$500 \mu \mathrm{m}$ & 2.6 & 2.2 & 2.4 & 2.6 \\
\hline & Fiducial & AGN-10x & & \\
$\mathrm{L}_{\mathrm{IR}}(8-1000 \mu \mathrm{m})$ & 2.5 & 14.4 & & \\
$\mathrm{~L}_{\mathrm{FIR}}(40-500 \mu \mathrm{m})$ & 2.0 & 7.2 & & \\
$\mathrm{~L}(10-30 \mu \mathrm{m})$ & 6.7 & 142.7 & & \\
$\mathrm{~L}(100-1000 \mu \mathrm{m})$ & 1.5 & 3.2 & & \\
& & & & \\
& & & &
\end{tabular}

Note. Maximal boosting as a function of $\lambda_{\text {obs }}$ is derived from the redshifted AGN-10x SED considering all time stamps and viewing angles. Generally, these maximum values occur around the most extreme snapshot in our simulations, where $L_{\mathrm{AGN}} / L_{\mathrm{SF}}>10$, and should be interpreted as the maximum possible correction an IR SFR would require to accurately recover the true dust-enshrouded SFR under such admittedly extreme conditions.

Although the current consensus is that star formation dominates cold dust emission at $\lambda \gtrsim 100 \mu \mathrm{m}$ (see Section 1), some empirical studies have suggested that AGNs play an important role in powering dust emission even at these wavelengths (e.g., Sanders \& Mirabel 1996). For example, Symeonidis et al. (2016), Symeonidis (2017), and Symeonidis \& Page (2018) show that optical through submillimeter emission in $z<0.18$ QSOs and $z \sim 1-2$ extremely luminous IR galaxies can be dominated by AGN-heated dust out to kiloparsec scales in the host galaxy; however, the degree of contribution by AGNs to $L_{\mathrm{IR}}$ for spatially unresolved systems can depend on assumptions made during SED modeling. Different methods find opposite conclusions based on the shape of the input AGN template SED in the FIR, as demonstrated in Stanley et al. (2017), who find negligible AGN contribution to submillimeter flux densities for $z \lesssim 0.5$ QSOs. For the simulations presented in this work, $L_{\mathrm{IR}}$ and $L_{\mathrm{FIR}}$ can be boosted at maximum by factors of $\sim 10$ and $\sim 7$, respectively, by AGN-powered cold dust emission (Table 1), which lends qualitative support to observational results favoring higher AGN contributions to FIR/submillimeter fluxes. This effect may lead to overestimates of IR-derived SFRs by a factor of $\sim 2-3$ on average for the most extreme cases of AGN-heated dust at $z \lesssim 3$ in galaxies with high $\lambda_{\text {edd }}$ and $L_{\mathrm{AGN}} / L_{\mathrm{SF}}>1$. Spectroscopic SFR diagnostics that do not depend on dust heating (e.g., [Ne II], [Ne III]) are better suited for measuring accurate SFRs in such cases (e.g., Ho \& Keto 2007); however, these lines can still suffer from significant attenuation in the mid-IR in extremely obscured cases (Smith et al. 2007).

For the reasons mentioned above, indicators of dustobscured AGN activity are especially important when attempting to measure coeval stellar and SMBH mass assembly. X-ray observations can identify Compton-thick QSOs in dusty galaxies out to high redshifts (e.g., Brandt \& Alexander 2015 and references therein). For example, using Chandra observations, Vito et al. (2020) discovered highly obscured AGNs residing within two dusty, star-forming galaxies resident in a $z \sim 4$ protocluster, one of which is a Compton-thick QSO that has a luminosity comparable to the most luminous QSOs 


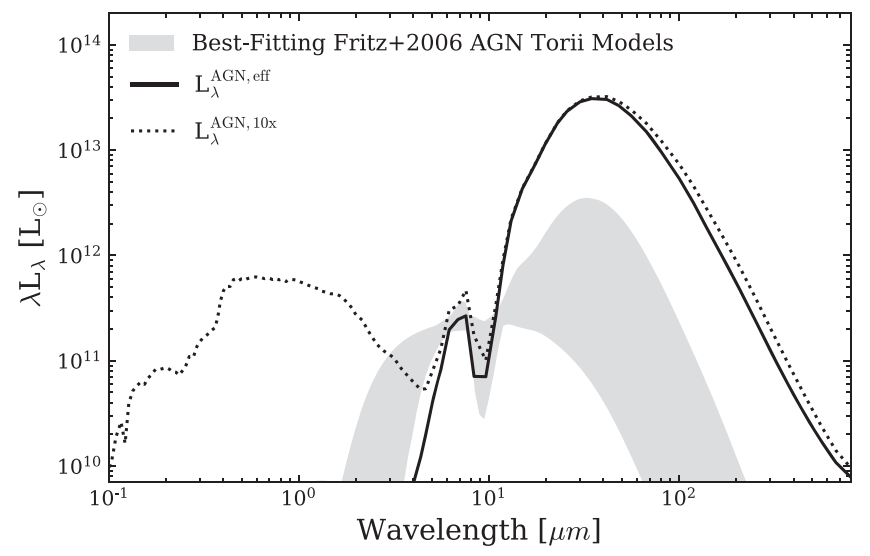

Figure 7. The total (dotted black line) and effective AGN (solid black line) SEDs already shown in Figure 1, but now compared against the dusty AGN torus models from Fritz et al. (2006). We fit the entire model library to the AGN-10x spectrum between 5 and $13 \mu \mathrm{m}$ and show the domain of the top 100 best-fitting models as a shaded gray region. The torus model can reproduce our effective AGN SED's mid-IR dust emission from $\sim 2$ to $10 \mu \mathrm{m}$ but drastically underestimates the FIR emission powered by the AGN. Thus, inferring the AGN contribution to the FIR using standard torus models would result in overestimating the SFR.

known. The results presented in the present work suggest that these obscured AGNs may power an appreciable fraction of the cold dust emission from these galaxies. This example demonstrates the value of X-ray follow-up of systems purportedly harboring high levels of obscured star formation. Alternatively, spectroscopic methods in the mid-IR $(\sim 3-30 \mu \mathrm{m})$ may be used to discriminate between dustenshrouded AGNs and star formation using high-excitation emission lines (Spinoglio \& Malkan 1992) or polycyclic aromatic hydrocarbon (PAH) features + mid-IR continuum decomposition (e.g., Pope et al. 2008; Kirkpatrick et al. 2015). Currently, mid-IR spectroscopic techniques are the most sensitive method for identifying dust-enshrouded AGNs at all levels of AGN strength relative to the luminosity of the host galaxy (Hickox \& Alexander 2018). Using, among other simulations, the fiducial run discussed in this work, Roebuck et al. (2016) find the fraction of $8-1000 \mu \mathrm{m}$ luminosity attributed to AGNs (the IR AGN fraction) to be a good predictor of the bolometric AGN fraction at times up to coalescence and at $A_{V}>1$. For such dust-obscured AGN phases, quantifying the AGN fraction using mid-IR data may enable subtracting host-galaxy AGN-heated dust emission to derive accurate SFRs for AGN hosts (e.g., Kirkpatrick et al. 2017).

Spectroscopic methods and spatially resolved data for more galaxies will improve our understanding of obscured mass assembly; however, a revision of SED-fitting techniques is necessary to fully leverage the power of future observatories. In particular, our results suggest that in highly dust-obscured galaxies the host-galaxy-scale dust emission cannot be considered decoupled from emission from the AGN, as is done in community-standard SED-fitting codes. As we show in Figure 7, simpler IR SED decomposition methods also suffer from the lack of AGN-heated cold dust, as it is common to subtract an AGN torus model from the FIR SED of a galaxy and assume that the residual power arises from star formation alone. If we make this assumption for our simulation and subtract the best-fitting Fritz et al. (2006) AGN torus model from the total SED, then the residual IR emission would overestimate the true SFR by a factor of $\sim 7$ in the extreme case where $L_{\mathrm{AGN}} / L_{\mathrm{SF}}$ is maximal. SED fits that include an AGN may also need to consider an associated FIR cold dust component that does not contribute to the inferred SFR.

\subsection{Limitations of This Work}

We have presented a "case study" of a single galaxy merger simulation. As emphasized above, the simulation is representative of $z \sim 2-3$ massive, rapidly star-forming, highly dustobscured galaxies (SMGs), and we thus expect our results to only apply to the bright end of the IR luminosity function. It would be valuable to repeat our analysis using simulations that span a much broader range of properties and redshifts.

As discussed in previous works (Younger et al. 2009; Hayward et al. 2011; Snyder et al. 2013), the treatment of subgrid dust structure in the ISM is a key uncertainty in RT calculations such as those used here. For the same set of simulations and RT calculations discussed in our work, Snyder et al. (2013) investigate the effects of varying the prescription for subgrid dust structure on the time-dependent SED. Notably, they compare the fiducial ISM model adopted in our work, which assumes a uniform dust distribution across each resolution element, with a "multiphase" prescription that assigns some time-dependent fraction of the gas to dense, cold clouds ( $\sim 27 \%$ at $\left.t_{\text {peak }}\right)$. In the multiphase model, attenuation and emission from the (subgrid) dense cold clouds are ignored ( i.e., it is assumed that they have negligible volume filling factor). They find that in the highly obscured regime, the FIR luminosity is insensitive to this assumption, and we have confirmed that our results are not qualitatively affected by the treatment of subgrid dust clumpiness. Nevertheless, a key avenue for future work is to repeat this analysis on simulations with much higher resolution and more sophisticated treatments of star formation, BH accretion, and stellar and AGN feedback (e.g., Angles-Alcazar et al. 2021). Such an analysis would enable us to check whether our conclusion that dustenshrounded AGNs can dominate host-galaxy-scale cold dust emission holds true for significantly more realistic simulations.

\section{Summary and Conclusions}

We have analyzed synthetic SEDs of a hydrodynamical galaxy merger simulation generated via dust RT calculations to investigate the influence heavily dust-enshrouded luminous AGNs may have on host-galaxy, kiloparsec-scale cold dust emission. Our main conclusions are as follows:

1. Heavily dust-enshrouded AGNs may power significant cold dust FIR emission on host-galaxy (kiloparsec) scales; in this particular extreme simulation, the AGN can boost the total IR luminosity by an order of magnitude and the FIR (100-1000 $\mu \mathrm{m})$ luminosity by a factor of 3 (i.e., the AGN can be the primary heating source of cold dust).

2. Our simulations have FIR colors longward of the Wien peak of dust emission consistent with those of $z \sim 2$ dusty galaxies, including AGN hosts and "purely star-forming" galaxies. The FIR colors of our simulations tracing the dust peak are somewhat warmer than observed galaxies when $L_{\mathrm{AGN}} / L_{\mathrm{SF}}$ is high. However, in both regimes, the FIR colors of the AGN-powered dust emission are redder than those of any of the widely used AGN torus SED models that we considered. 
3. Our results may have significant consequences for IRand (sub)millimeter-selected galaxies, which are more likely to feature significant host-galaxy obscuration of the AGN they host than are less dust-obscured galaxies. For such systems, if a deeply dust-enshrouded AGN is present, the FIR emission may be powered by both young stars and the AGN. Applying standard torus models, which do not include host-galaxy-scale AGN-powered cold dust emission, to decompose the SED may thus result in overestimating the SFR and underestimating the AGN fraction.

This work should be considered a "case study" that demonstrates the need for caution when interpreting the FIR SEDs of galaxies that may host dust-enshrouded AGNs. Spatially resolved observations (from, e.g., ALMA and JWST) and additional AGN diagnostics should be sought to confirm whether a dust-enshrouded AGN is present when studying such systems. Moreover, there is a need to develop AGN SED models that account for dust obscuration and emission from both the clumpy torus and the host galaxy rather than treating the AGN and its host as decoupled.

We thank the anonymous referee, M. Symeonidis, K. Phadke, and E. Daddi for insightful comments on the manuscript, which helped us significantly improve this work. The Flatiron Institute is supported by the Simons Foundation. H.A.S. and J.R.M.-G. acknowledge partial support from NASA grants NNX14AJ61G and NNX15AE56G and from SOFIA grant NNA17BF53C (08_0069). L.R. acknowledges the SAO REU program, funded by the National Science Foundation REU and Department of Defense ASSURE programs under NSF grant AST-1659473 and by the Smithsonian Institution. The simulations in this paper were performed on the Odyssey cluster supported by the FAS Research Computing Group at Harvard University. This research has made use of NASA's Astrophysics Data System.

\section{ORCID iDs}

Jed McKinney ๑ https://orcid.org/0000-0002-6149-8178 Christopher C. Hayward (1) https://orcid.org/0000-00034073-3236

Lee J. Rosenthal (1) https://orcid.org/0000-0001-8391-5182 Alexandra Pope (1) https://orcid.org/0000-0001-8592-2706 Anna Sajina (1) https://orcid.org/0000-0002-1917-1200

\section{References}

Aird, J., Nandra, K., Laird, E. S., et al. 2010, MNRAS, 401, 2531 Angles-Alcazar, D., Quataert, E., Hopkins, P., et al. 2021, ApJ, 917, 53 Azadi, M., Aird, J., Coil, A. L., et al. 2015, ApJ, 806, 187 Banerji, M., Jones, G. C., Wagg, J., et al. 2018, MNRAS, 479, 1154 Begelman, M. C. 2002, ApJL, 568, L97

Bower, R. G., Benson, A. J., Malbon, R., et al. 2006, MNRAS, 370, 645 Brandt, W. N., \& Alexander, D. M. 2015, A\&ARv, 23, 1

Cañameras, R., Nesvadba, N., Kneissl, R., et al. 2017, A\&A, 604, A117

Carilli, C. L., \& Walter, F. 2013, ARA\&A, 51, 105

Casey, C. M., Narayanan, D., \& Cooray, A. 2014, PhR, 541, 45

Chang, Y.-Y., Le Floc'h, E., Juneau, S., et al. 2017, ApJS, 233, 19

Cicone, C., Maiolino, R., Sturm, E., et al. 2014, A\&A, 562, A21

Ciesla, L., Charmandaris, V., Georgakakis, A., et al. 2015, A\&A, 576, A10 Croton, D. J., Springel, V., White, S. D. M., et al. 2006, MNRAS, 365, 11 de Grijp, M. H. K., Lub, J., \& Miley, G. K. 1987, A\&AS, 70, 95 Delvecchio, I., Gruppioni, C., Pozzi, F., et al. 2014, MNRAS, 439, 2736 Di Matteo, T., Springel, V., \& Hernquist, L. 2005, Natur, 433, 604 Dietrich, J., Weiner, A. S., Ashby, M. L. N., et al. 2018, MNRAS, 480, 3562
DiMascia, F., Gallerani, S., Behrens, C., et al. 2021, MNRAS, 503, 2349

Downes, D., \& Solomon, P. M. 1998, ApJ, 507, 615

Ferrarese, L., \& Merritt, D. 2000, ApJL, 539, L9

Franceschini, A., Braito, V., Persic, M., et al. 2003, MNRAS, 343, 1181

Fritz, J., Franceschini, A., \& Hatziminaoglou, E. 2006, MNRAS, 366, 767

Gebhardt, K., Bender, R., Bower, G., et al. 2000, ApJL, 539, L13

Genzel, R., Burkert, A., Bouché, N., et al. 2008, ApJ, 687, 59

Genzel, R., Lutz, D., Sturm, E., et al. 1998, ApJ, 498, 579

Gürkan, G., Hardcastle, M. J., Jarvis, M. J., et al. 2015, MNRAS, 452, 3776

Hatziminaoglou, E., Omont, A., Stevens, J. A., et al. 2010, A\&A, 518, L33

Hayward, C. C., Jonsson, P., Kereš, D., et al. 2012, MNRAS, 424, 951

Hayward, C. C., Kereš, D., Jonsson, P., et al. 2011, ApJ, 743, 159

Hayward, C. C., Narayanan, D., Kereš, D., et al. 2013, MNRAS, 428, 2529

Hayward, C. C., Torrey, P., Springel, V., Hernquist, L., \& Vogelsberger, M. 2014, MNRAS, 442, 1992

Hickox, R. C., \& Alexander, D. M. 2018, ARA\&A, 56, 625

Ho, L. C., \& Keto, E. 2007, ApJ, 658, 314

Hopkins, P. F., Hernquist, L., Cox, T. J., \& Kereš, D. 2008, ApJS, 175, 356

Hopkins, P. F., Richards, G. T., \& Hernquist, L. 2007, ApJ, 654, 731

Jiang, Y.-F., Stone, J. M., \& Davis, S. W. 2014, ApJ, 796, 106

Jonsson, P. 2006, MNRAS, 372, 2

Jonsson, P., Groves, B. A., \& Cox, T. J. 2010, MNRAS, 403, 17

Kalfountzou, E., Stevens, J. A., Jarvis, M. J., et al. 2014, MNRAS, 442, 1181

Kelly, B. C., \& Shen, Y. 2013, ApJ, 764, 45

Kereš, D., Katz, N., Fardal, M., Davé, R., \& Weinberg, D. H. 2009, MNRAS, 395,160

Kereš, D., Katz, N., Weinberg, D. H., \& Davé, R. 2005, MNRAS, 363, 2

Kirkpatrick, A., Pope, A., Alexander, D. M., et al. 2012, ApJ, 759, 139

Kirkpatrick, A., Pope, A., Sajina, A., et al. 2015, ApJ, 814, 9

Kirkpatrick, A., Pope, A., Sajina, A., et al. 2017, ApJ, 843, 71

Kormendy, J., \& Ho, L. C. 2013, ARA\&A, 51, 511

Lanzuisi, G., Delvecchio, I., Berta, S., et al. 2017, A\&A, 602, A123

Leja, J., Johnson, B. D., Conroy, C., \& van Dokkum, P. 2018, ApJ, 854, 62

Madau, P., \& Dickinson, M. 2014, ARA\&A, 52, 415

Massardi, M., Enia, A. F. M., Negrello, M., et al. 2018, A\&A, 610, A53

Nandra, K., \& Iwasawa, K. 2007, MNRAS, 382, L1

Nelson, D., Pillepich, A., Springel, V., et al. 2018, MNRAS, 475, 624

Nenkova, M., Sirocky, M. M., Ivezić, Z. `, \& Elitzur, M. 2008a, ApJ, 685, 147

Nenkova, M., Sirocky, M. M., Nikutta, R., Ivezić, Ž., \& Elitzur, M. 2008b, ApJ, 685, 160

Page, M. J., Stevens, J. A., Mittaz, J. P. D., \& Carrera, F. J. 2001, Sci, 294, 2516

Petric, A. O., Ho, L. C., Flagey, N. J. M., \& Scoville, N. Z. 2015, ApJS, 219, 22

Pope, A., Chary, R.-R., Alexander, D. M., et al. 2008, ApJ, 675, 1171

Pouliasis, E., Mountrichas, G., Georgantopoulos, I., et al. 2020, MNRAS, 495,1853

Ramos Padilla, A. F., Ashby, M. L. N., Smith, H. A., et al. 2020, MNRAS, 499, 4325

Roebuck, E., Sajina, A., Hayward, C. C., et al. 2016, ApJ, 833, 60

Ruiz, A., Carrera, F. J., \& Panessa, F. 2007, A\&A, 471, 775

Sajina, A., Yan, L., Armus, L., et al. 2007, ApJ, 664, 713

Sanders, D. B., \& Mirabel, I. F. 1996, ARA\&A, 34, 749

Sanders, D. B., Phinney, E. S., Neugebauer, G., Soifer, B. T., \& Matthews, K. 1989, ApJ, 347, 29

Sanders, D. B., Soifer, B. T., Elias, J. H., Neugebauer, G., \& Matthews, K. 1988, ApJL, 328, L35

Schneider, R., Bianchi, S., Valiante, R., Risaliti, G., \& Salvadori, S. 2015, A\&A, 579, A60

Scoville, N., Murchikova, L., Walter, F., et al. 2017, ApJ, 836, 66

Shangguan, J., Ho, L. C., Bauer, F. E., Wang, R., \& Treister, E. 2020, ApJ, 899,112

Shankar, F., Weinberg, D. H., \& Miralda-Escudé, J. 2009, ApJ, 690, 20

Sharda, P., Federrath, C., da Cunha, E., Swinbank, A. M., \& Dye, S. 2018, MNRAS, 477, 4380

Shirakata, H., Kawaguchi, T., Oogi, T., Okamoto, T., \& Nagashima, M. 2019, MNRAS, 487, 409

Smith, J. D. T., Draine, B. T., Dale, D. A., et al. 2007, ApJ, 656, 770

Snyder, G. F., Hayward, C. C., Sajina, A., et al. 2013, ApJ, 768, 168

Spinoglio, L., \& Malkan, M. A. 1992, ApJ, 399, 504

Springel, V. 2005, MNRAS, 364, 1105

Springel, V., Di Matteo, T., \& Hernquist, L. 2005, MNRAS, 361, 776

Springel, V., \& Hernquist, L. 2003, MNRAS, 339, 289

Stacey, H. R., McKean, J. P., Robertson, N. C., et al. 2018, MNRAS, 476,5075 
Stanley, F., Alexander, D. M., Harrison, C. M., et al. 2017, MNRAS, 472, 2221

Swinbank, A. M., Dye, S., Nightingale, J. W., et al. 2015, ApJL, 806, L17 Symeonidis, M. 2017, MNRAS, 465, 1401

Symeonidis, M., Giblin, B. M., Page, M. J., et al. 2016, MNRAS, 459, 257

Symeonidis, M., \& Page, M. J. 2018, MNRAS, 479, L91

Tacconi, L. J., Genzel, R., Neri, R., et al. 2010, Natur, 463, 781
Vito, F., Brandt, W. N., Lehmer, B. D., et al. 2020, A\&A, 642, A149

Wethers, C. F., Banerji, M., Hewett, P. C., \& Jones, G. C. 2020, MNRAS, 492,5280

Yang, G., Boquien, M., Buat, V., et al. 2020, MNRAS, 491, 740

Younger, J. D., Hayward, C. C., Narayanan, D., et al. 2009, MNRAS, 396, L66 Yun, M. S., \& Scoville, N. Z. 1998, ApJ, 507, 774 